International Journal of Engineering \& Technology, $7(1.5)(2018) 217-220$
SPC
International Journal of Engineering \& Technology
Website: www.sciencepubco.com/index.php/IJET
Research paper

\title{
Bigdata implementation of apriori algorithm for handling voluminous data-sets
}

\author{
Dr. M. Nagalakshmi ${ }^{1 *}$, I. Surya Prabha ${ }^{2}$, K. Anil ${ }^{3}$ \\ ${ }^{I}$ Associate Professor, Dept of CSE Marri Laxman Reddy Institute of technology \& management \\ ${ }^{2}$ Professor Dept of IT Institute of Aeronautical Engineering \\ ${ }^{3}$ Assistant Professor Dept of CSE Marri Laxman Reddy Institute of technology \& management \\ *Corresponding author E-mail: nagalakshmimalempati@gmail.com
}

\begin{abstract}
Apriori is one all instructed the key algorithms to come again up with frequent itemsets. Analysing frequent itemset could be an critical step in analysing based info and recognize association dating among matters. This stands as degree standard basis to supervised gaining knowledge of, that encompasses classifier and feature extraction strategies. making use of this system is vital to grasp the behaviour of structured data. maximum of the dependent information in scientific domain square measure voluminous. method such moderately info desires country of the artwork computing machines. setting up region such degree infrastructure is high priced. so a allotted environment admire a clustered setup is hired for grappling such situations. Apache Hadoop distribution is one all advised the cluster frameworks in allotted environment that enables by means of distributing voluminous data across style of nodes most of the framework. This paper specializes in map/reduce trend and implementation of Apriori formula for dependent info analysis.
\end{abstract}

Keywords: Frequent Itemset, Distributed Computing,Hadoop, Apriori, Distributed data processing

\section{Introduction}

In many applications of the $\$ 64000$ global, generated statistics is of satisfactory challenge to the neutral due to it gives you purposeful facts / information that assists in making sibyllic analysis. this records helps in enhancing certain choice parameters of the applying that modifications the general final results of a commercial enterprise approach. the range of know-how, vicinity along mentioned as factsunits, generated through the making use of is implausibly big. So, there's a demand of approach massive records-units expeditiously. The records-set accrued might also also be from heterogeneous sources and can be based or unstructured facts. technique such facts generates useful patterns from that facts are going to be extracted. the best approach is to use this instance and insert headings and textual content into it as relevant. statistics processing is that the tactic of finding correlations or patterns among fields in massive facts-units and build up the knowledge-base, supported the given constraints. the goal of informationof recordsof understanding mining is to extract information from degree current statistics-set and retread it into a human-understandable shape for any use. This technique is typically referred to as records Discovery in information-units (KDD). the tactic has revolutionized the method of finding the state-of-the-art real-international troubles. KDD technique consists of series of obligations like alternative, preprocessing, transformation, facts processing and interpretation as proven in Figure1.

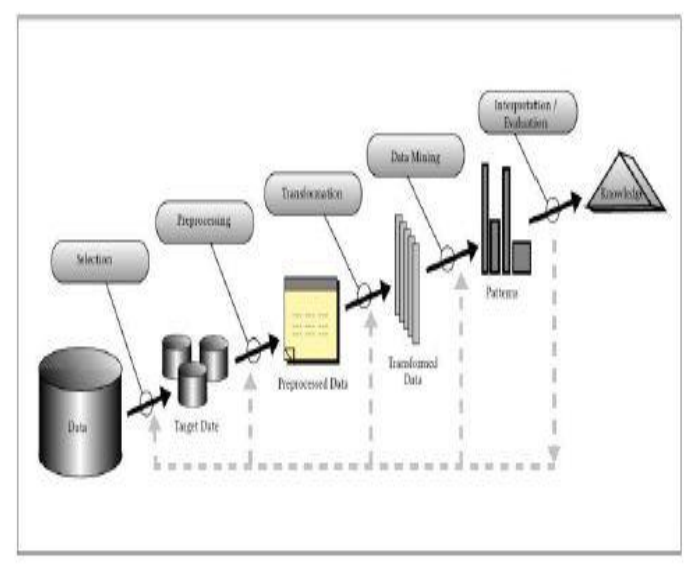

Fig. 1: KDD Process

In a dispensed computing surroundings is also a bunch of loosely coupled method nodes connected through community. each nodes contributes to the execution or distribution / replication of statistics. it is found as a cluster of nodes. There rectangular degree numerous methods of fitting a cluster, certainly one of it truly is generally discovered as cluster framework. Such frameworks implement the installing area method and replication nodes for statistics. Examples region unit DryAdLinq and Apache Hadoop (also cited as Map / 
reduce). the alternative strategies involve installing region of cluster nodes on advert-hoc foundation and not being nice with the aid of a rigid framework. Such strategies merely involve a group of API calls essentially for remote method invocation (RMI) as a area of interprocedure conversation. Examples square degree Message Passing Interface (MPI) and a version of MPI cited as MPJExpress. the method of putting in vicinity a cluster depends upon the information densities and on top of factors the eventualities indexed beneath:

- The facts is generated at numerous locations and needs to be accessed locally most of the time for approach.

- The data and technique is sent to the machines in the cluster to reduce the impact of any precise device being overloaded that damages its approach

This paper is organized as follows, future phase will discuss touching on to finish survey of the connected work distributed the domain of the distributed processing, specifically focused on finding frequent object units. The section 3 of this paper discusses concerning the layout and implementation of the Apriori rule tuned to the dispensed environment, preserving a key target the experimental test-mattress demand. The section four, discusses referring to the effects of the check setup supported Map/reduce - Hadoop. ultimately finish our paintings with the phase five.

\section{Connected work}

dispensed processing in Peer-to-Peer Networks (P2P) [1] offers partner in Nursing outline of disbursed information-mining applications and algorithms for peer-to-peer environments. It describes every real partner in Nursingd approximate allotted factsmining algorithms that process in an exceptionally decentralised manner. It illustrates those methods for the matter of computing and remark clusters inside the understanding dwelling on the numerous nodes of a peer-to-peer network. This paper focuses on partner rising department of allotted processing brought up as peer-to-peer processing. It collectively gives a pattern of exact and approximate P2P algorithms for cluster in such dispensed environments. net provider-based method for processing in disbursed environments [2] provides partner method to expand a records mining machine in distributed environments. This paper offers an online carrierprimarily based approach to get to the bottom of those issues. The machine is created practice this approach gives a normal presentation and garage mechanism, platform freelance interface, and a dynamically extensible style. The projected approach throughout this paper allows users to categorise new incoming facts by selecting one in all the previously learnt models. design for processing in disbursed environments [3] describes machine style for climbable and portable dispensed processing packages. This method offers a record parent of speech brought up as emph for accessing and attempting to find virtual documents in stylish dispensed statistics systems. The paper describes a corpus linguistic analysis of enormous textual content corpora supported co places with the purpose of extracting linguistics family members from unstructured textual content. allotted processing of big Classifier Ensembles [4] gives a replacement classifier mixture strategy that scales up effectively and achieves each excessive prognostic accuracy and trait of issues with excessive exceptional. It induces a global model by way of studying from the averages of the local classifiers output. The effective combination of huge fashion of classifiers is executed this fashion. Multi Agent-primarily based allotted processing [5] is that the mixing of multi-agent machine and allotted processing (MADM), collectively delivered up as multi-agent primarily based distributed processing. the attitude right here is in phrases of significance, system define, existing systems, and analysis traits. This paper gives associate in Nursing define of MADM systems that ar prominently in use. It conjointly defines the common elements between systems and affords a pinnacle level view in their approaches wherein and design. protecting privacy and Sharing the facts in allotted setting practice cryptographic approach on hot and bothered statistics [6] proposes a framework that permits systematic transformation of unique records exercise abnormal statistics perturbation approach. The modified statistics is then submitted to the machine via cryptographic approach. this approach is relevant in allotted environments in which each information proprietor has his very own data and wishes to share this with the alternative knowledge householders. At equal time, this statisticsthis factsthis facts owner desires to keep the privacy of touchy understanding inside the facts. disbursed anonymous information perturbation method for privatenessmaintaining processing [7] discusses a light-weight anonymous information perturbation method for low-cost privateness retaining in distributed processing. a couple of protocols ar projected to handle these constraints and to guard understanding facts and conjointly the company technique towards collusion attacks.An formula for frequent pattern Mining supported Apriori[8] proposes three fully definitely different frequent pattern mining tactics (record clear out, Intersection and conjointly the projected set of rules) supported classical Apriori algorithmic rule. This paper plays a comparative look at of all three tactics on a information-set of 2000 transactions. This paper surveys the listing of current association rule mining strategies and compares the algorithms with their modified approach. using Apriori-like algorithms for Spatio-Temporal sample Queries [9] offers the handiest way to assemble Apriori-like algorithms for mining spatio-temporal patterns. This paper addresses issues of the numerous varieties of scrutiny capabilities which may be accustomed mine frequent patterns.Map-reduce for device getting to know on Multi core [10] discusses methods in which at some stage in which to increase a commonly applicable parallel programming paradigm that is applicable to fully absolutely exceptional mastering algorithms. with the aid of taking gain of the summation type at some stage in a map-reduce framework, this paper attempts to put an oversized vary of machine getting to know algorithms and reach an full-size acceleration on a twin processor cores. mistreatment Spot times for MapReduce paintings flows [11] describes new techniques to boost the runtime of MapReducejobs. This paper offers Spot times (SI) as a manner of accomplishing overall performance profits at low economic value.

\section{Style and implementation}

\subsection{Experimental setup}

The experimental setup has three nodes linked to controlled switch joined to private LAN. one altogether these nodes is prepared as Hadoop master or as a result of the call node that controls the facts distribution over the Hadoop cluster. all of the nodes place unit equal in terms of the device configuration i.e., all of the nodes have equal processor - Intel Core2 couple and assembled via conventional manufacturer. As inquiring attempt, configuration created to understand Hadoop could have three nodes in completely distributed mode. The aim is to scale the amount of nodes by way of mistreatment traditional cluster control package package deal which can simply upload new nodes to Hadoop rather than putting in Hadoop in every node. The visual photograph of this setup is proven a few of the discern 2 . 


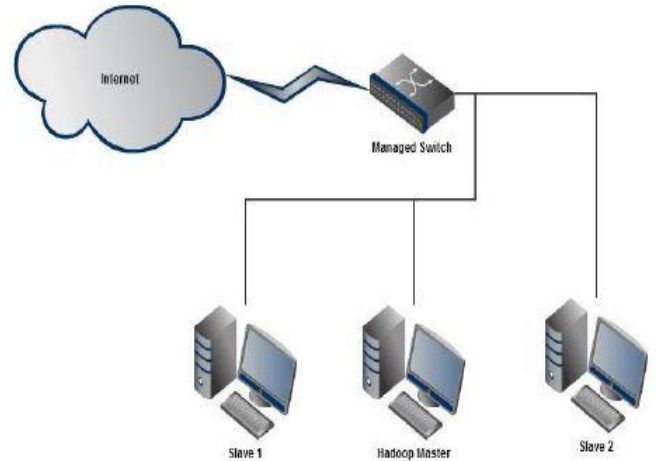

Fig. 2: Experimental Setup for Hadoop Multi-node

\subsection{System readying}

The standard hobby of the desired gadget is unreal the use of the device organization as portrayed in the determine three. series of Map calls is shaped to send the facts to cluster node and conjointly the format is of the shape <Key, cost> then a cut back calls is implemented to summarize the consequent from complete absolutely special nodes. a straightforward program is up to expose these consequences to person operational the Hadoop master

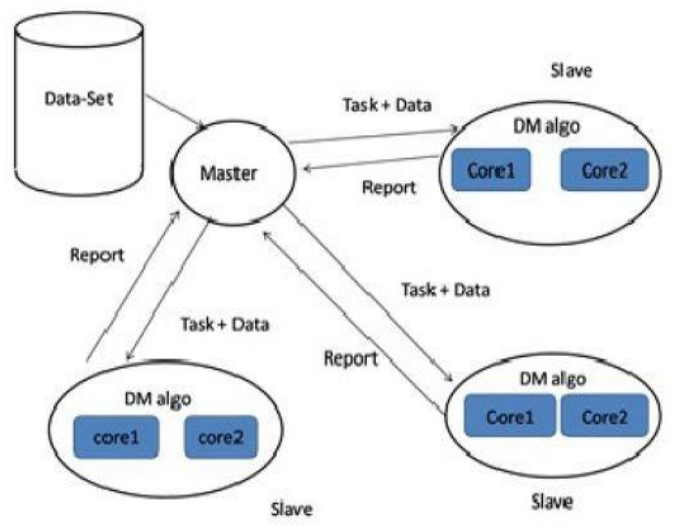

Fig. 3: System Organization 3.3 Algorithm style

The components stated produces all of the subsets a good way to be generated from the given object set. any those subsets ar searched in opposition to the records-sets and for this reason the frequency is mentioned. There or innumerable info things and their subsets, thence they need to be searched them at same time in order that seek time reduces. as a result, the Map-lessen idea of the Hadoop fashion comes into picture. Map perform is forked for every set of the objects. those maps can run on any node in the disbursed placing prepared underneath Hadoop configuration. the undertaking distribution is taken care by means of the Hadoop system and thus the documents, information-sets required ar area into HDFS. In every Map function, the rate is that the item set. the full of the informationset is scanned to go looking out the entry of the fee item set and consequently the frequency is referred to. that is regularlythis could be regularly given as diploma output to the dimensions returned operate in the dimensions back magnificence printed inside the Hadoop center bundle. in the reducer function, every output of the each map is amassed and it is region into wanted record with its frequency. system is referred to below in herbal language:

\section{Results}

The experimental setup pictured earlier than has been rigorous examined towards a Pseudo-dispensed configuration of Hadoop and with standalone computer for variable intensity of information and act. The altogether prepared multi-node Hadoop with differential gadget configuration (FHDSC) would take relatively terribly whilst to technique information as in opposition to the altogether prepared similar multi-nodes (FHSSC)). Similarity is in terms of the gadget configuration starting from portable laptop style to bundle running in it. this may be surely pictured within the discern four.

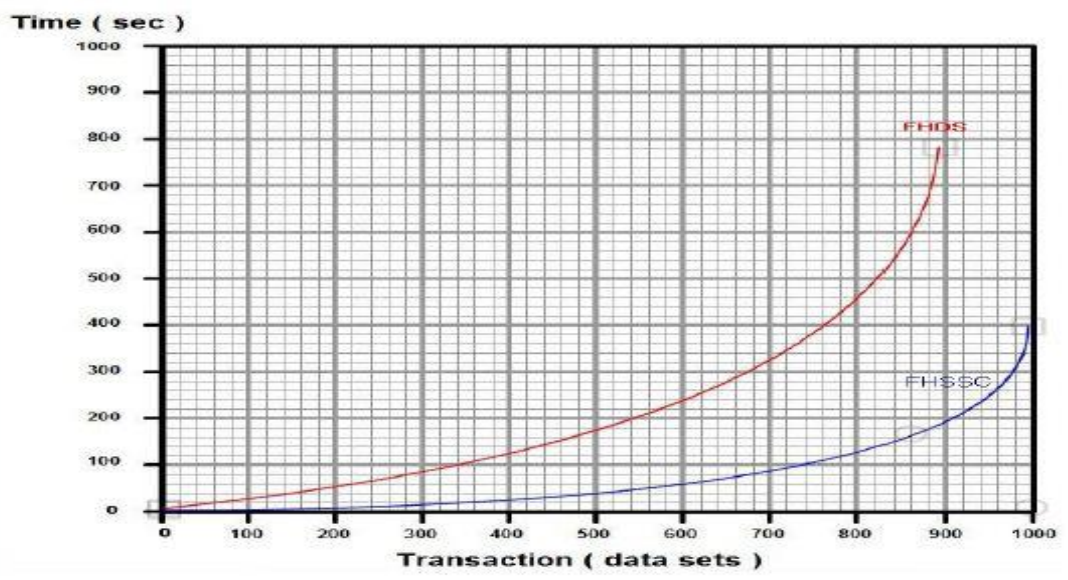

Fig. 4: FHDSC Vs. FHSSC 
The results for taken from the 3-node Fully-distributed and Pseudo distributed modes of Hadoop for large dealings area unit fairly smart until it reaches the utmost threshold capability of nodes. The result is delineate within the figure five.

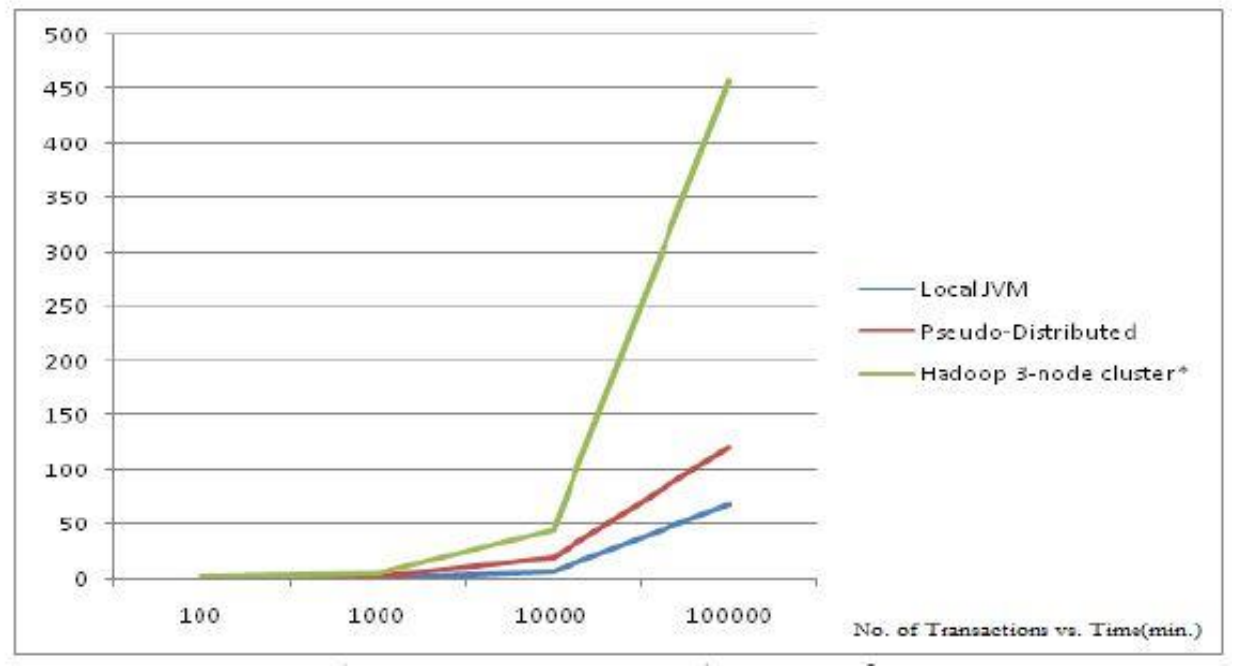

Fig. 5: Transactions VsHadoop Configuration

looking the graph, there's large variance in time visible at threshold of twelve, 000 transactions. past that the time is in exponential. this can be thanks to the computer design and limited garage capability of $80 \mathrm{~GB}$ in step with Node. thence the superset dealings generation can take longer time to calculate and consequently the mineworker for frequent item-set. wherever $\mathrm{N}$ is that the range of nodes put in within the cluster.

\section{Conclusions and future enhancements}

The paper gives a totally extraordinary technique of fashion algorithms for clustered placing. this can be applicable to situations as soon as there data-in depth computation is required. Such setup presents a broad road for investigation and analysis in processing. making an try the demand for such algorithmic rule there's pressing should be compelled to attention and discover heaps of regarding clustered putting specially for this domain.

\section{References}

[1] Souptik Datta, Kanishka Bhaduri, Chris Giannella, Ran Wolff, and Hillol Kargupta, Distributed DataMining in Peer-to-Peer Networks, Universityof Maryland, Baltimore County, Baltimore, MD, USA,

[2] Journal IEEE Internet Computing archive Volume 10 Issue 4, Pages 18 - 26, July 2006

[3] Ning Chen, Nuno C. Marques, and Narasimha Bolloju, A Web Service based approach for data mining in distributed environments, Department of Information Systems, City University of Hong Kong, 2005

[4] Mafruz Zaman A shrafi, David Taniar, and Kate A. Smith, A Data Mining Architecture for Distributed Environments, pages 27-34, Springer-Verlag London, UK, 2007.

[5] Grigorios Tsoumakas and Ioannis Vlahavas, Distributed Data Mining of Large Classifier Ensembles, SETN-2008, Thessaloniki, Greece, Proceedings, Companion Volume, pp. 249-256, 11-12 April 2008.

[6] Vuda Sreenivasa Rao, Multi Agent-Based Distributed Data Mining: An Over View, International Journal of Reviews in computing, pages 83-92,2009.

[7] P.Kamakshi, A.Vinaya Babu, Preserving Privacy and Sharing the Datain Distributed Environment using Cryptographic Technique on
Perturbed data, Journal Of Computing, Volume 2, Issue 4, ISSN 21519617, April2010.

[8] Feng LI, Jin MA, Jian-hua LI, Distributed anonymous data perturbation method for privacy-preserving data mining, Journal of Zhejiang University SCIENCE A ISSN 1862-1775, pages 952-963, 2008.

[9] Goswami D.N. et. al., An Algorithm for Frequent Pattern Mining Based On Apriori (IJCSE) International Journal on Computer Science and Engineering Vol. 02, No. 04, 942-947, 2010.

[10] Marcin Gorawski and Pawel Jureczek, Using Apriori-like Algorithms for Spatio-Temporal Pattern Queries, Silesian University of Technology, Institute of Computer Science, Akademicka 16, Poland, 2010

[11] Cheng-Tao Chu et. al., Map-Reduce for Machine Learning on Multicore, CS Department, Stanford University, Stanford, CA, 2006.

[12] Navraj Chohanet. al., See Spot Run: Using Spot Instances for MapReduce Workflows, Computer Science Department, University of California,2005.

[13] Rajesh, M., and J. M. Gnanasekar. \&quot;Congestion control in heterogeneous wireless ad hoc network using FRCC.\&quot; Australian Journal of Basic and Applied Sciences 9.7 (2015): 698702.

[14] S.V.Manikanthan and V.Rama"Optimal Performance Of Key Predistribution Protocol In Wireless Sensor Networks" International Innovative Research Journal of Engineering and Technology ,ISSN NO: 2456-1983,Vol-2,Issue -Special -March 2017.

[15] T. Padmapriya and V. Saminadan, "Inter-cell Load Balancing Technique for Multi- class Traffic in MIMO - LTE - A Networks", International Conference on Advanced Computer Science and Information Technology, Singapore, vol.3, no.8, July 2015. 